\title{
The Effects of Classical Music based Chakra Meditation on the Symptoms of Premenstrual Syndrome
}

\author{
Poornima Viswanathan ${ }^{1}$, Nishal Pinto ${ }^{2}$
}

\section{ABSTRACT:}

Premenstrual syndrome (PMS) is a very common condition, occurring in women of reproductive age. This is an important source of stress for women, significantly affecting their regular functioning. Previous studies have shown that alternative forms of therapy like meditation have an effect on the symptoms associated with PMS. However, the direct link between the two has not been focused on by the larger group of researchers. In this study, the researcher intended to study the effects of classical music based chakra meditation on the symptoms of premenstrual syndrome. The sample of the study comprised of 40 college students who were screened for premenstrual syndrome based on the Shortened Premenstrual Assessment Form (SPAF) and Calendar of Premenstrual Experiences(COPE) which they were expected to fill in for one menstrual cycle. The participants who qualified for the study were allotted to the intervention group and control group. The intervention group was expected to practice a 15 minute music based chakra meditation, everyday, for one menstrual cycle, during which they also filled in the COPE. The control group was expected to fill the COPE for one menstrual cycle. Both the groups filled in the SPAF after the completion of the menstrual cycle. The results on the SPAF and COPE showed that there was significant reduction in all the post-test symptom clusters of the experimental group, compared with the pre-test symptoms. Thus, this study demonstrates that music based chakra meditation can have significant healing effects on the symptoms of premenstrual syndrome.

Keywords: premenstrual syndrome, chakra meditation, carnatic music

Premenstrual syndrome has always been a topic closely associated with controversy, since it is a condition that affects such a large amount of people, thus inciting offended reactions when termed as a 'disorder'. When viewed as a disorder, many women who suffer from this problem have hopes of getting it treated and being relieved of the suffering (Keep \& Utian, 1981).

\footnotetext{
${ }^{1}$ Student, Clinical Psychology, Christ University, Bangalore, Karnataka

${ }^{2}$ Assistant Professor, Clinical Psychology, Christ University, Bangalore, Karnataka
} 
However, this topic has been subject to inadequate research, and in spite of the experimentation of various treatment techniques, none of the methods have proven effective enough to be standardized or established. Another cause for controversy is that the very definition has no clarity or consensus (Connolly,2001).

The symptoms of premenstrual syndrome are mainly emotional, although at times they come in a physical form. Like the name suggests, there are almost 200 symptoms that constitute this syndrome (Pfaff, 2002). The most frequent of these symptoms are bloating, weight gain, fatigue, energy loss, headaches, cramps, joint and muscle aches, low back pain, breast swelling and tenderness, cravings for sweet and/or salty food, increased or decreased sleeping hours, reduced sex drive, constipation or diarrhoea, sadness and depression, anger, irritability, aggression, anxiety, mood swings, decreased alertness, concentration trouble and withdrawal from family and friends (Pfaff, 2002).

Like previously mentioned, PMS has no established treatments (Moe,1998). Recommendations usually range from mild exercises and controlled intake of caffeine; while more severe cases demand the need for medications like antidepressants and SSRIs (Premenstrual Syndrome(PMS) -Treatment, 2013). There are, however, a few alternative forms of healing. Owing to the fact that PMS's emotional symptoms consist of mainly mood swings, the usual treatments that clinicians and therapists prescribe are relaxation techniques (Kotsirilos, Vitetta \& Sali, 2011). The most widely used of these are deep breathing exercises and progressive muscle relaxation (Atreya, 2014).

In India, meditation is one of the most commonly used relaxation techniques. This technique is used to increase the mind's feelings of love and peace, powerful positive feelings that increase happiness and relaxation (Weiss, 2013). Emerging as a very effective treatment from this area is the Chakra meditation. It works on the concept that there exist three nadis(energy channels) and seven chakras( energy centres) within every human being, and all of these have to be in balance (Saradananda, 2008). Decoding and overseeing the state of all these parts is accomplished by getting to the root of this system and the link between all of these chakras. The root is the creative, called Kundalini, dormant energy that powers the full system. This spreads to each chakra through the spinal column and culminates at the top of the head (Bean,2014). Through the Chakra meditation, the person learns to pick apart and see with clarity the state of their inner system, also gaining the ability to diagnose and correct it (Bean, 2014).

It has been hypothesised that that PMS and other menstruation-related problems are born from the imbalance of energy centres - or chakras - in our body, and restoration of the balance can be done using Chakra meditation (“Chakra System”,n.d.).

One highly efficient form of chakra meditation is based on music and ragas (Music therapy for meditation, n.d.). Here, different chakras are associated with different ragas of carnatic music, and each is played simultaneous to the individual focussing and concentrating on each corresponding body part whose chakra is associated to the raga being played (Redmond,2004). 
Each of the seven basic musical notes in the musical octave, or swaras, have a one-to-one correspondence with each of the seven chakras (Healing by Music Therapy - Ragas ,2004). Likewise, in a manner that corresponds with these swaras, certain ragas as well play a part in activating certain chakras ("Music and Chakras", n.d.)

Thus, since it is proven that classical music, by itself contains numerous properties of relaxation (Nayar,2012); and chakra meditation is also highly helpful in elimination of anxiety symptoms and mood disturbances; a combination of the two might prove to be a highly effective way of minimising the symptoms of PMS.

\section{METHOD}

\section{Statement of the Problem}

To identify the effect of classical music based chakra meditation on symptoms of premenstrual syndrome.

\section{Operational Definitions}

Premenstrual syndrome. Premenstrual syndrome can be defined as a consistent pattern of physical and emotional symptoms that occur during the luteal phase of the menstrual cycle and cause significant interference in daily life activities.

Music based chakra meditation. Music based chakra meditation can be defined as the process of bringing about a balance in the different chakras of the body by focusing on the different ragas and the corresponding body parts related to each chakra.

\section{HYPOTHESIS}

- There is no significant difference in the severity of symptoms of premenstrual syndrome of the participants in the intervention group before and after the intervention on the shortened premenstrual assessment form.

- There is no significant difference in the severity of symptoms of premenstrual syndrome of the participants in the intervention group before and after the intervention on the total score on the daily calendar

- There is no significant difference in the severity of symptoms of premenstrual syndrome of the participants in the control group between the first and second administration of the shortened premenstrual assessment form.

- There is no significant difference in the severity of symptoms of premenstrual syndrome of the participants in the intervention group before and after the intervention on the total score on the daily calendar. 


\section{Research Design}

A quantitative paradigm was used in the study. A quasi-experimental design which involves both pre-test post-test design as well as an experimental and control group was used to study the effect of the intervention on the symptoms of premenstrual syndrome.

\section{Sample}

The sample chosen for the study was 40 female students in the age group of 18 to 23years with symptoms of premenstrual syndrome. The sample was chosen based on convenience sampling by approaching college students from different branches of study that showed a consistent pattern of premenstrual symptoms based on the Shortened Premenstrual Assessment form(SPAF) which was an online form. Initially 65 students filled in the SPAF, from which 52 students were chosen for the second stage - filling the Calendar of Premenstrual Experiences (COPE). Out of these participants, 45 were selected for the next phase and 23 were assigned to the intervention group and 22 to the control group. The final number of participants who successfully completed the study was 40 .

\section{Tools Used}

Shortened premenstrual assessment form(SPAF). The Shortened Premenstrual Assessment Form by Allen, McBride and Pirie (1991) was used for the basic screening of participants to see if they experienced any significant premenstrual symptoms.

Calendar of premenstrual experiences(COPE). After selection of participants using the SPAF, they were expected to record their daily experience of symptoms using the Calendar of Premenstrual Experiences constructed by Mortola, Girton, Beck and Yen(1991) for a period of one month.

\section{Procedure}

Female college-going students were approached by the researcher for participation in the study through online social networks and bringing it to the notice of different colleges in Bangalore and Chennai. Female students were asked whether they experienced changes in their emotions and physical conditions in relation to their menstrual cycle. These students were given the link to the online SPAF prospective participants were identified based on the pattern of symptoms. These participants were requested to record their daily symptoms through one menstrual cycle in the COPE and mail it to the researcher after a month. This report of symptoms was analysed to identify participants who experienced consistent patterns of premenstrual symptoms, only during the luteal phase of the menstrual cycle. The participants were enrolled for the study based on their interest and availability. 45 participants were be selected and 23 participants were assigned to the intervention group and 22 to the control group respectively. Participants whose menstrual phase fell around the same time of the month were included so that the intervention can be given accordingly. Informed consent was be obtained from the participants. The intervention 
consisted of the participants listening to an audio tape which consists of a 15 minute recording of music based guided chakra meditation which was formulated in consultation with an expert. The intervention group was required to do the guided meditation for three days a week for a period of one month when the researcher monitored them through Skype, and they were also be given a copy of the recording, so that they practiced the meditation at home on the other four days of the week. This was followed up through phone calls and messages. The intervention began on the first day of the menstrual cycle and ended on the first day of the next cycle. The participants recorded their symptoms everyday during this one month on the COPE and at the end of one month, they were given the SPAF. The control group was not given any intervention. However they completed the COPE and SPAF.

\section{Data Analysis}

Paired sample t-test was used to compare the pre-test and post-test data.

\section{RESULTS AND DISCUSSION}

\section{Results}

Table 1 showing the results of paired sample t test of pre-test and post-test score on SPAF of the intervention group

\begin{tabular}{|l|l|l|l|l|l|l|}
\hline Variable & Administration & $\mathrm{N}$ & Mean & SD & $\mathrm{t}$ & Sig \\
\hline Total SPAF & Pre-test & 20 & 32.25 & 6.77 & 9.28 & .000 \\
\hline & Post test & 20 & 19.80 & 5.14 & & \\
\hline
\end{tabular}

There is a significant difference between the pre-test $(M=32.25$, S.D.=6.77) and post-test scores $(\mathrm{M}=19.80, \mathrm{~S} . \mathrm{D} .=5.14)$ on the SPAF of the intervention group; $\mathrm{t}=9.28, \mathrm{p}=0.000$

Table 2 showing the results of paired sample t test of pre-test and post-test total score of the intervention group on the calendar of premenstrual experiences

\begin{tabular}{|l|l|l|l|l|l|l|}
\hline Variable & Administration & $\mathrm{N}$ & Mean & SD & $\mathrm{t}$ & Sig \\
\hline Total & Pre-test & 20 & 151.80 & 35.25 & 9.10 & .000 \\
\hline & Post test & 20 & 88.20 & 24.30 & & \\
\hline
\end{tabular}

There is a significant difference between the pre-test $(M=151.80$, S.D. $=35.25)$ and post-test scores $(M=88.20$, S.D. $=24.30)$ on the total score of the intervention group. $t=9.10, p=0.000$ 
The Effects of Classical Music based Chakra Meditation on the Symptoms of Premenstrual Syndrome

Table 3 showing the results of paired sample t test of first and second administration score on SPAF of the control group

\begin{tabular}{|l|l|l|l|l|l|l|}
\hline Variable & Administration & $\mathrm{N}$ & Mean & SD & $\mathrm{t}$ & Sig \\
\hline Total SPAF & Pre-test & 20 & 33.10 & 6.73 & .32 & .76 \\
\hline & Post test & 20 & 31.50 & 6.78 & & \\
\hline
\end{tabular}

There is no significant difference between the first month $(M=33.10$, S.D.=6.73) and second month scores $(M=31.50$, S.D. $=6.78)$ on the SPAF of the intervention group, $t=.32, p=0.76$

Table 4 showing the results of paired sample t test of first and second month on total score of the control group on the calendar of premenstrual experiences

\begin{tabular}{|l|l|l|l|l|l|l|}
\hline Variable & Administration & $\mathrm{N}$ & Mean & SD & $\mathrm{t}$ & Sig \\
\hline Total & Pre-test & 20 & 129.45 & 43.07 & -4.72 & .64 \\
\hline & Post test & 20 & 131.75 & 42.97 & & \\
\hline
\end{tabular}

There is a no significant difference between the first month $(\mathrm{M}=129.45$, S.D.=43.07) and second month $(\mathrm{M}=131.75$, S.D. $=42.97)$ on the total score of the intervention group, $\mathrm{t}=--4.72, \mathrm{p}=0.642$

\section{DISCUSSION}

The purpose of the present research was to study the effectiveness of music based chakra meditation on the symptoms of premenstrual syndrome. The results of the study indicate that Chakra meditation has had a significant effect on the intervention group, leading to a reduction in the symptoms of premenstrual syndrome. This is indicated by the analysis based on the scores on the Shortened Premenstrual Assessment Form and Calendar of Premenstrual experiences.

Table1 shows that the pre-test scores of the participants in the intervention group on the Shortened Premenstrual Assessment Form is higher than the post-test scores, indicating a reduction in the severity of symptoms after the intervention. Based on this, the hypothesis which states that there is no significant difference in the severity of symptoms of premenstrual syndrome of the participants in the intervention group before and after the intervention on the shortened premenstrual assessment form is rejected. Hence, based on these results, a conclusion can be drawn that chakra meditation has a significant effect on the reduction of symptoms of premenstrual syndrome. Meditation results in a state of mind which promotes the well-being of the individual who practices it, and results in a sense of peace. At the same time, chakra meditation is a form that involves focusing on different body parts and increasing awareness about the functioning of these organs, thereby bringing about a balance in the functioning of the entire system (Bean, 2014). These effects of meditation and regular and focused practice result in the reduction of symptoms of premenstrual syndrome which involve both physiological and 
psychological symptoms. There are a few studies which may provide explanation for the research for the present study. A review study conducted by Arias, Steinberg, Banga and Trestman(2006) showed that meditation techniques have an effect on different illnesses, including premenstrual syndrome. Similarly, a study performed by Konandreas and Kolokithas (1989) showed that biofeedback and relaxation have a positive effect on mood states during the luteal phase of the menstrual cycle.

The effects of chakra meditation are also demonstrated by Table 4 which shows that the pre-test scores are higher than the post-test scores for the intervention group on the Calendar of Premenstrual Experiences. This leads to the conclusion that the intervention has led to a reduction of symptoms and has a significant effect, thus resulting in the rejection of the hypothesis which states that there is no significant difference severity of symptoms of premenstrual syndrome of the participants in the intervention group before and after the intervention on the total score on the daily calendar. These results are supported by a study conducted by Goodale, Domar and Benson(1990) on the alleviation of symptoms of premenstrual syndrome through the relaxation response. This study also used similar scales, which are the Premenstrual Assessment Form and Daily Rating Form, and it was seen that out of the three groups, the relaxation group experienced the highest benefits. Since meditation is also a kind of relaxation response, this study can offer support to the results of the present study.

An analysis was made on the specific symptoms of premenstrual syndrome based on the Calendar of Premenstrual Experiences, to check for the effect of Chakra meditation on these symptoms. The symptom clusters assessed for included Fluid retention, appetite, cognitive/autonomic and emotional symptoms. The results showed that Chakra meditation has a significant effect on the appetite related symptoms, on comparison of the pre-test and post-test scores of the intervention group and the post-test scores of the intervention and control group.

Previous studies showed that charting and recording the symptoms of premenstrual syndrome also have an effect on the reduction of symptoms. The study done by Goodale, Domar and Benson(1990) showed that there was a seventeen percent reduction of symptoms in the charting group. Hence, to rule out the effects of recording symptoms on the Calendar of Premenstrual Experiences, the present study used a control group which did not go through the intervention, but recorded their symptoms on the calendar for the entire menstrual cycle. It was hypothesized that there would be no significant difference in the severity of symptoms of premenstrual syndrome of the participants in the control group between the first and second administration of the SPAF and between the first and second month on the total score on the daily calendar. According to Table 5 and Table 8, both the hypotheses are accepted as there is no significant difference between the first and second administration on the control group, and hence shows that the recording of symptoms has not had an effect on reducing the symptoms.

Thus, an overall conclusion can be drawn from the results that the intervention group experienced a significant reduction in the premenstrual symptoms on practising a chakra meditation intervention for 15 minutes every day for a period of one month. 


\section{CONCLUSIONS}

Thus the hypotheses which stated that there is no significant difference between the pre-test and post-test scores of the intervention group on the SPAF, and the calendar of premenstrual experiences were rejected. The hypotheses which stated that there is no significant difference between the pre and post-test scores of the control group on the SPAF and the calendar of premenstrual experiences were rejected. The hypotheses which stated that there would be no difference between the intervention and control group on the post-test scores of both these tools were also rejected. Thus, it can be concluded from the results of the present study that chakra meditation has a significant effect in reducing the number and severity of symptoms of individuals with symptoms of premenstrual syndrome.

\section{IMPLICATIONS}

The study implies that individuals, who suffer from symptoms of PMS which affect their regular functioning, can practise this form of meditation to bring them relief from the symptoms. As it requires only 15 minutes a day, it can also be practised by busy professionals. It can be used by therapists in a premenstrual clinic or a general physician as a recommendation for symptom relief. This finding opens up avenues for new research studies in this area which can focus on the effects of chakra meditation on more severe conditions associated with menstruation such as dysmenorrhoea and premenstrual dysphoric disorder. The present study studied the effects of the intervention only for a period of one month; future studies can focus on the long-term effects of practising chakra meditation on premenstrual symptoms.

\section{LIMITATIONS}

However, there are a few limitations to the present study. The study was done only on 40 participants selected through convenience sampling, with only 20 in the intervention group. Hence, the generalization of the results to the entire population is under question. The participants were only monitored directly through Skype for only three days a week and for the rest of the four days, they were monitored through phone calls and messages, and their word was taken for the practice of the intervention. Future studies can be conducted on a larger sample and can be directly monitored every day.

\section{REFERENCES}

1. Allen, S. S., McBride, C. M., \& Pirie, P. L. (1991). The shortened premenstrual assessment form. The journal of reproductive medicine, 36(11), 769-772. Retrieved from http://europepmc.org/abstract/med/1765953.

2. Arias, A. J., Steinberg, K., Banga, A., \& Trestman, R. L. (2006). Systematic Review of the Efficacy of Meditation Techniques as Treatments for Medical Illness. Journal of Alternative and Complementary Medicine, 12(8). doi:10.1089/acm.2006.12.817

3. Atreya. (2014). Ayurvedic healing for women:Herbal gynecology. New Delhi: Motilal Banarsidass. 
4. Bean, G. A. (2014). The essentials of meditation. United States of America : Author

5. Chakra meditation for deep healing and balancing. (n.d.). Retrieved December 2, 2014, from WellBeing Alignment: http://www.wellbeingalignment.com/chakrameditation.html

6. Chakra System - Your Spiritual Library, Sunshine Healing Arts. (n.d.). Retrieved from http://www.sunshinehealingarts.net/the-chakras/

7. Connolly, M. (2001). Premenstrual syndrome: an update on definitions, diagnosis and management. Advances in Psychiatric Treatment. doi:10.1192/apt.7.6.469

8. Goodale, I. L., Domar, A. D., \& Benson, H. (1990). Alleviation of premenstrual syndrome symptoms with the relaxation response. Behavioral Medicine, New England Deaconnes Hospital, 75(4). Retrieved from http://www.massgeneral.org/bhi/assets/pdfs/publications/goodale\%201990\%20obstet\%2 0gynecol.pdf

9. Healing by Music Therapy - Ragas - (Akhandjyoti). (2004, September). Retrieved August 5, 2014, from http://www.akhandjyoti.org/?Akhand-Jyoti/2004/SeptOct/MusicTherapyRagas/

10. Keep, V., \& Utian, W. (1981). The Premenstrual Syndrome. United States: Springer Science.

11. Konandreas., \& Kolokithas, G. (1989). The effect of biofeedback and relaxation on premenstrual syndrome. Pace University.

12. Kotsirilos, V., Vitetta, L., \& Sali, A. (2011). A guide to evidence-based integrative and complementary medicine. Australia: Elsevier.

13. Moe, B. A. (1998). Coping with PMS (premenstrual syndrome). New York: Rosen Publ.

14. Mortola, J., Girton, L., Beck, L., \& Yen, S. (1991). Diagnosis of premenstrual syndrome by a simple, prospective, and reliable instrument: The calendar of premenstrual experiences.International Journal of Gynecology \& Obstetrics. doi:10.1016/00207292(91)90644-K.

15. Music and Chakras - Shankar Ramani, Carnatic Vocal Musician \& Teacher in New Jersey (n.d.). Retrieved from http://www.shankarramani.com/music-and-chakras.html

16. Nayar, U. S. (2012). Power of music to alleviate academic stress in adolescents. In Child and adolescent mental health (pp. 227-238). New Delhi, India: Sage Publications.

17. Pfaff, W. (2002). Hormones, Brain and Behavior. USA: Elsevier Science.

18. Premenstrual Syndrome(PMS) -Treatment. (2013, December 2). Retrieved November 2014, from NHS choices: http://www.nhs.uk/Conditions/PremenstrualSyndrome/Pages/Treatment.aspx

19. Redmond, L. (2004). Chakra meditation: Transformation through the seven energy centers of the body. Boulder, CO: Sounds True.

20. Saradananda, S. (2008). Chakra Meditation: Discover Energy, Creativity, Focus, Love, Communication, Wisdom and Spirit. London: Duncan Baird Publishers.

21. Weiss, G. (2013). The healing power of meditation. Basic Health Publications. 